\title{
Morbidity and Older Persons' Perceptions of the Quality of Their Primary Care
}

\author{
Efrat Shadmi, RN, PhD, ${ }^{*}$ Cynthia M. Boyd, MD, MPH, ${ }^{* \dagger}$ Chun-Ju Hsiao, MHS, * \\ Martha Sylvia, RN, MSN, MBA, Alyson B. Schuster, MPH, MBA,* and \\ Chad Boult, MD, MPH, MBA*'
}

OBJECTIVES: To quantify the association between community-dwelling older persons' level of morbidity and their perceptions of the quality of their primary care.

DESIGN: Cross-sectional study.

SETTING: Urban primary care practice.

PARTICIPANTS: A sample of community-dwelling members of a capitated health plan aged 65 and older who were patients of four general internists in one urban practice $(\mathrm{N}=120)$.

MEASUREMENTS: The Johns Hopkins Adjusted Clinical Groups case-mix system was used to designate a person's aggregate morbidity level as moderate or high depending on the number and types of chronic conditions they had. Aspects of quality of primary care (physician-patient communication, interpersonal treatment, knowledge of patient, integration of care, and trust in physician) were assessed using the Primary Care Assessment Survey.

RESULTS: All participants were classified as having moderate $(41 \%)$ or high morbidity $(59 \%)$. Older adults with high morbidity assigned a lower rating to all aspects of quality of primary care (physician-patient communication $(P=.001)$, interpersonal treatment $(P=.002)$, knowledge of patient $(P=.03)$, integration of care $(P=.004)$, and trust in physician $(P=.01))$ than those with moderate morbidity. The differences in quality of primary care remained statistically significant after controlling for age, sex, race, and education level.

CONCLUSION: Older persons with multiple chronic conditions report inadequate quality of primary care and dissatisfaction with their care. Those with high morbidity levels experience poorer quality of primary care than those

From the * Department of Health Policy and Management, Bloomberg School of Public Health, and ${ }^{\dagger}$ Division of Geriatric Medicine and Gerontology and Center on Aging and Health, Department of Medicine, School of Medicine, Johns Hopkins University, Baltimore, Maryland; and ${ }^{\ddagger}$ Johns Hopkins HealthCare, LLC, Glen Burnie, Maryland.

Some of the data from this study were presented at the American Geriatrics Society 2004 annual meeting as a poster presentation.

Address correspondence to Efrat Shadmi, Haifa University, Mount Carmel, Haifa, Israel 31905. E-mail: eshadmi@univ.haifa.ac.il

DOI: $10.1111 /$ j.1532-5415.2005.00578.x with moderate morbidity in all five aspects measured here. J Am Geriatr Soc 54:330-334, 2006.

Key words: quality; primary care; multiple morbidity

M eeting the healthcare needs of older persons with multimorbidity is one of the most daunting challenges facing U.S. health care today. The majority of Medicare beneficiaries aged 65 and older $(65 \%)$ have more than one chronic condition and receive most of their care from primary care physicians. ${ }^{1,2}$ These physicians strive to address their patients' multiple coexisting problems, attempting to follow evidence-based guidelines (some of which conflict $\left.{ }^{3,4}\right)$; prioritizing treatments according to patients' wishes; and integrating the efforts of specialists, hospitals, home care agencies, and other providers. Medicare does not compensate for most of the time physicians devote to communication and coordination. ${ }^{5}$ Thus, ensuring that multimorbid older persons receive high-quality chronic care may be difficult. Evidence suggesting that multimorbidity may diminish quality of care is just beginning to emerge. Breakdowns in coordination of care and potentially preventable hospital admission for conditions for which hospitalization is thought to be avoidable with the application of good primary care have been reported to occur frequently in older persons with chronic conditions. ${ }^{1,6}$

The aim of this study was to quantify the association between community-dwelling older persons' morbidity levels and the quality of their primary health care. Diagnoses from insurance claims were used to classify each person's morbidity level, and a mailed survey was conducted to assess each person's perceptions of the quality of their care.

\section{METHODS}

\section{Study Sample}

A cross-sectional design was used to quantify the association between patients' morbidity and the quality of their primary care. The data were collected during a baseline assessment of high-risk participants in a pilot study of an experimental model of chronic care (Guided Care). 
The population from which the sample was drawn consisted of all 861 community-dwelling members of a capitated health plan aged 65 and older who were patients of four general internists in one urban practice. A validated predictive model (Adjusted Clinical Groups Predictive Model (ACG-PM)) was applied to 12 months of this population's insurance claims to select the $20 \%$ who were at highest risk for generating extreme health insurance expenditures during the following year. The ACG-PM uses age, sex, and International Classification of Disease, Ninth Revision (ICD-9), diagnoses to estimate the high-risk probability scores. ${ }^{7}$ After persons who were already participating in other case management or care coordination programs were excluded, 150 high-risk older persons were eligible to participate in the study.

A mailed survey was conducted to measure the quality of care that each participant received. Each eligible person received a preliminary letter from the health plan, followed by a packet containing a cover letter, a questionnaire, a stamped return envelope, and a $\$ 1$ bill. Nonrespondents were called and asked to complete the survey by completing and returning the questionnaire or answering the questions by telephone.

\section{Questionnaire}

The questionnaire inquired about health status, functional ability, demographic characteristics, and components of the Primary Care Assessment Survey (PCAS). ${ }^{8}$ The PCAS is a validated instrument that asks patients to rate 11 essential aspects of the quality of their primary care, as defined by the Institute of Medicine. ${ }^{9}$ Five of the 11 scales and two individual items of the PCAS were included in this study:

Physician-Patient Communication: thoroughness of questions about symptoms, attention to what the patient says, clarity of explanations and instructions, help in making decisions.

Interpersonal Treatment: patience, friendliness, caring, respect, time spent with patient.

Knowledge of Patient: medical history, responsibilities at home or work, principal health concerns, values, and beliefs.

Integration of Care: coordination and synthesis of specialty care and/or care while hospitalized.

Trust of Physician: integrity, competence, advocacy.

Organizational Access (2 items): Time needed to reach physician's office by phone;

Time needed to reach physician by phone.

Respondents rated each PCAS item on a 6-point scale, ranging from very poor to excellent. A score for each PCAS scale was computed by summing the scores of its individual items and normalizing the total to a range of 0 to 100 . The PCAS, which has been used with other populations of older adults, ${ }^{10}$ provides a comprehensive measure of patients' experience of each of the five domains assessed in this study. For example, the PCAS assesses three important elements of care coordination: the facilitation of patients' access to specialty care, communication with specialists, and the integration of care received from different sources. ${ }^{11}$

In addition, respondents rated their health status on a 5 -point scale (poor to excellent) and reported difficulty in performing activities of daily living (bathing, dressing, eat- ing, transferring, and toileting $\left.{ }^{12}\right)$ and instrumental activities of daily living (preparing meals, taking medications, performing housework, using the telephone, managing money, walking across a room, getting to places beyond walking distance, and shopping $\left.{ }^{13}\right)$. The questionnaire also included items about satisfaction with health care (5-point scale, very dissatisfied to very satisfied) and adequacy of follow-up after visits to specialists.

\section{Morbidity}

The ACG case mix software was used to measure each participant's level of morbidity. ${ }^{7}$ In this algorithm, every ICD-9 code belongs to a group of conditions that usually require similar amounts of health care. The ACG software designates a person as having an aggregate morbidity level of 1 to 6 (low to high), depending on the number and type of such groups populated by the ICD-9 codes on 12 months of recent insurance claims for the person's health care.

In addition, to be able to describe the types and number of health conditions of the study population, 12 months of recent health insurance claims were used, and the prevalence of nine common chronic conditions among the respondents were estimated: hypertension, ischemic heart disease, osteoarthritis, diabetes mellitus, congestive heart failure, chronic obstructive pulmonary disease, depression, dementia, and Parkinson's disease. The Johns Hopkins Bloomberg School of Public Health institutional review board approved the study.

\section{Data Analysis}

All statistical analyses were performed using Stata statistical software, Version 8 (Stata Corp., College Station, TX). Bivariate analyses were conducted using chi-square tests for categorical variables and $t$ tests for continuous variables to determine whether persons with moderate morbidity differed from those with high morbidity with regard to their demographic characteristics, self-rated health status, functional ability, satisfaction with primary care, and quality of primary care. Next, a multivariate linear regression model of the relationship between morbidity level and each of five aspects of the quality of care was constructed, adjusting for age, race, sex, and education level. These covariates have been shown in other studies to affect older persons' reports of quality of care. ${ }^{10,14}$ The distribution of residuals showed that the data fit all five models well.

\section{RESULTS}

Of the 150 high-risk older persons surveyed, 120 (80\%) responded. The ACG case-mix software classified all of the respondents as having moderate or high morbidity. Their demographic, health-related, functional, and morbidity characteristics are shown in Table 1. Of the 120 respondents, $10 \%$ had a diagnosis of dementia. The diagnosis of dementia was derived from insurance claims, which do not describe severity. Because all surveyed persons lived at home, it was presumed that any dementia was not severe. In addition, the responses of proxies were accepted by allowing help from another person whom the respondent described as a personal, informal caregiver, because proxies could be expected to observe the quality domains measured by the PCAS. ${ }^{15}$ 
Table 1. Characteristics of Study Sample $(\mathrm{N}=120)$

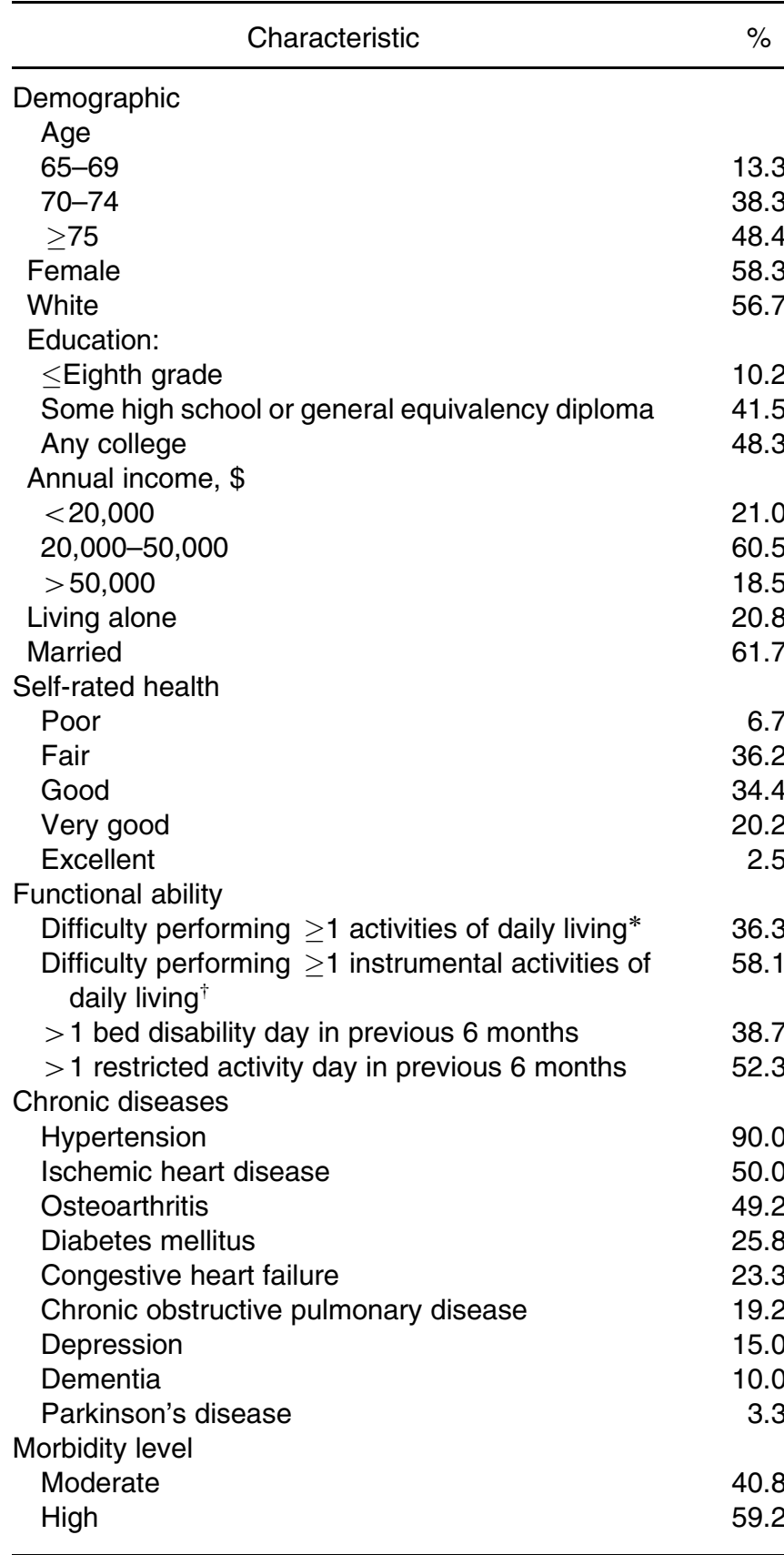

* Bathing, dressing, eating, getting in or out of a chair, toileting.

${ }^{\dagger}$ Using the telephone, doing housework, taking medications, getting to places beyond a walking distance, preparing meals, shopping, and managing money.

Table 2 presents bivariate comparisons of respondents with high and moderate morbidity. Respondents with high morbidity assigned lower ratings to all five measured aspects of the quality of their primary care (physicianpatient communication $(P=.001)$, interpersonal treatment $(P=.002)$, knowledge of patient $(P=.003)$, integration of care $(P=.004)$, and trust of physician $(P=.01))$ than those with moderate morbidity. Persons with high morbidity were also significantly less likely to be very satisfied with their overall care $(53 \%)$ than those with moderate morbidity $(79 \%)(P=.004)$. As shown in Table 3, all of these differences in the quality of primary care were statistically significant in multivariate linear regression models that adjusted for the effects of age, sex, race, and education level.

\section{DISCUSSION}

This study found that older persons with high morbidity report lower quality of primary care than those with moderate morbidity. This effect was consistent across five important domains of quality: communication, interpersonal treatment, knowledge, integration, and trust. The relationship between patients' burden of morbidity and their perceived quality of primary care may be related to the complexity of their health care. That is, patients with several chronic conditions often experience fast-paced visits as primary care physicians attempt to address all their medical needs. They often also receive care from several specialty physicians. Providing effective coordination of care and communication is challenging, and the primary care physician's questions, examinations, and instructions may seem superficial, rushed, tense, and impersonal.

The quality of health care is a multidimensional construct that encompasses several technical and interpersonal attributes. The results reported here add to emerging literature on the quality of primary care for older persons and the relationship between quality and the characteristics of the person receiving the care. Although older adults usually rate their care more positively than younger adults, there is heterogeneity within older adults regarding views of medical care, with those aged 80 and older less likely than then persons aged 65 to 69 to be very satisfied with the care they receive. ${ }^{14} \mathrm{~A}$ recent study of noninstitutionalized Medicare beneficiaries found substantial declines between 1998 and 2000 in financial access to care, visit-based continuity, and integration of care. ${ }^{10}$ Another study found that, among community-dwelling Medicare beneficiaries, those with disabilities were significantly more likely to report having poor communication with their physicians, as well as poor access to them. ${ }^{15,16}$

Satisfaction with care is likely to mediate the relationship between patients' morbidity and their ratings of the quality of their health care. That is, sicker patients may be less satisfied and rate their care as worse. In this study, respondents with high morbidity were less likely to report high satisfaction with care than were respondents with moderate morbidity. Previous studies have also reported an association between self-reported health status and satisfaction with care. ${ }^{17}$

Patients' ratings of the quality of their primary care have been shown to be associated with clinical outcomes. One study showed that physicians' knowledge of their patients and patients' trust in their physicians, as measured using the PCAS, were strongly associated with patients' adherence to physicians' advice. Furthermore, the leading correlates of self-reported improvements in health were physician-patient communication, integration of care, knowledge of the patient, trust of the physician, and thoroughness of examination. ${ }^{18}$ Another study of Medicare beneficiaries found that trust of the physician was associated with receipt of clinical preventive services, such as influenza vaccination and mammography. ${ }^{19}$

Aspects of the study design require judicious interpretation of the results reported here. All of the study 
Table 2. Characteristics of Respondents with Moderate and High Morbidity

\begin{tabular}{|c|c|c|c|}
\hline Characteristic & $\begin{array}{l}\text { Moderate Morbidity } \\
\qquad(\mathrm{n}=49)\end{array}$ & $\begin{array}{l}\text { High Morbidity } \\
\qquad(\mathrm{n}=71)\end{array}$ & $P$-value* \\
\hline \multicolumn{4}{|l|}{ Demographic } \\
\hline Age, mean $\pm S D$ & $74.3 \pm 6.2$ & $76.2 \pm 5.9$ & .045 \\
\hline Female, n (\%) & $30(61.2)$ & $40(56.3)$ & .59 \\
\hline White, $\mathrm{n}(\%)$ & $28(57.1)$ & $40(56.3)$ & .93 \\
\hline Some college, n (\%) & $23(47.9)$ & $34(48.6)$ & .94 \\
\hline Self-rated health status very good to excellent, $\mathrm{n}(\%)$ & $13(26.5)$ & $14(20.0)$ & .40 \\
\hline \multicolumn{4}{|l|}{ Functional ability, $n(\%)$} \\
\hline Difficulty performing any activity of daily living ${ }^{\dagger}$ & $12(26.6)$ & $29(42.6)$ & .08 \\
\hline Difficulty performing any instrumental activity of daily living & $22(53.6)$ & $39(60.9)$ & .46 \\
\hline \multicolumn{4}{|l|}{ Ratings of quality of primary care } \\
\hline \multicolumn{4}{|l|}{$\begin{array}{l}\text { Primary Care Assessment Survey domains, } \\
\text { mean } \pm \text { SD (range } 0-100)\end{array}$} \\
\hline Communication & $82.0 \pm 2.2$ & $72.2 \pm 2.3$ & .001 \\
\hline Interpersonal treatment & $84.7 \pm 2.2$ & $76.4 \pm 1.9$ & .002 \\
\hline Knowledge of patient & $75.5 \pm 2.5$ & $66.5 \pm 2.1$ & .003 \\
\hline Integration of care & $77.4 \pm 2.5$ & $68.5 \pm 2.2$ & .004 \\
\hline Trust of physician & $81.8 \pm 1.8$ & $76.4 \pm 1.5$ & .01 \\
\hline \multicolumn{4}{|l|}{ Access to care } \\
\hline Time to reach office by phone & $3.8 \pm 0.2$ & $3.2 \pm 0.2$ & .01 \\
\hline Time to reach physician by phone & $3.4 \pm 0.2$ & $2.9 \pm 0.2$ & .048 \\
\hline \multicolumn{4}{|l|}{ Follow-up care, $\mathrm{n}(\%)^{\mathcal{S}}$} \\
\hline Discussed specialty visit with primary care physician & $32(78.0)$ & $41(82.0)$ & .64 \\
\hline Knows whom to call with question about specialty visit & $39(95.1)$ & $45(91.8)$ & .53 \\
\hline Very satisfied with care, $\mathrm{n}(\%)^{\|}$ & $39(79.0)$ & $38(53.0)$ & .004 \\
\hline
\end{tabular}

* $P$-values derived from $t$ tests for continuous variables and chi-square tests for categorical variables.

† Bathing, dressing, eating, getting in or out of a chair, toileting.

${ }^{*}$ Using the telephone, doing housework, taking medications, getting to places beyond a walking distance, preparing meals, shopping, managing money.

$\S$ Of 97 respondents who reported seeing a specialist within the previous 6 months.

" Satisfaction with all medical care was rated on a scale of 1-5 (very dissatisfied to very satisfied).

$\mathrm{SD}=$ standard deviation

participants were older, high-risk, community-dwelling members of one capitated health plan who received their primary care from one practice. The four internists and 150 persons surveyed in this study might not be representative of all primary care physicians and high-risk older patients. It has been shown, for example, that for nine of the 11 original PCAS indicators, Medicare beneficiaries rated the quality of fee-for-service primary care more favorably than beneficiaries of health maintenance organizations. ${ }^{10} \mathrm{How}^{-}$ ever, findings from other larger studies in various other settings also indicate that poorer health is associated with lower ratings of the quality of health care. ${ }^{14-16}$ Furthermore, the cross-sectional design of this study precludes conclusions about causality.
In conclusion, this study illustrates the extant gap in quality of primary care of older persons with multiple, complex healthcare needs. Older persons with multiple chronic conditions report inadequate quality of primary care and greater dissatisfaction with their care, which is worse at higher morbidity levels. This may lead to poor clinical outcomes and unnecessary use of health care. Future studies should examine the effect of multimorbidity on objective indicators of quality, such as rates of duplications or omissions of tests as indicators of breakdowns in care coordination or rates of influenza vaccination as indicators of comprehensive care. Studies should also attempt to identify which forms and combinations of morbidity have the greatest effect on the quality of primary care. Clinicians and

\begin{tabular}{|c|c|c|c|c|c|}
\hline \multirow{2}{*}{$\begin{array}{c}\text { Primary Care } \\
\text { Assessment Survey } \\
\text { Scale (Range 0-100) }\end{array}$} & Communication & $\begin{array}{l}\text { Interpersonal } \\
\text { Treatment }\end{array}$ & $\begin{array}{l}\text { Knowledge } \\
\text { of Patient }\end{array}$ & $\begin{array}{l}\text { Integration } \\
\text { of Care }\end{array}$ & Trust \\
\hline & \multicolumn{5}{|c|}{$\beta$ Coefficient (Standard Error) } \\
\hline Intercept & $80.30(8.09)$ & $86.77(7.09)$ & $69.04(8.36)$ & $83.41(8.34)$ & $78.93(5.68)$ \\
\hline High morbidity & $-9.61(3.32)^{*}$ & $-8.11(2.91)^{*}$ & $-9.27(3.34)^{*}$ & $-8.92(3.35)^{*}$ & $-5.42(2.33)^{\dagger}$ \\
\hline
\end{tabular}

Note: Multiple linear regression adjusted for age, sex, race, and education level.

$* .01>P>.001$.

${ }^{\dagger} .05 \geq P \geq .01$. 
healthcare managers should continue to develop and test innovative models for providing high-quality primary care to multimorbid older persons, possibly through physician education, information technology, physician-nurse collaboration, and extended office visits.

\section{ACKNOWLEDGMENTS}

Johns Hopkins HealthCare provided support for this study. Johns Hopkins Bloomberg School of Public Health holds the copyright on the ACG case-mix system and receives royalties for its commercial use.

Financial Disclosure: All authors report that they have not received any financial support for this manuscript.

Author Contributions: Efrat Shadmi and Chad Boult: concept and design, acquisition of subjects and data, analysis, interpretation of data, preparation of manuscript. Cynthia Boyd: acquisition of subjects and data, analysis, interpretation of data, preparation of manuscript. Martha Sylvia, Chun-Ju Hsiao, and Alyson Schuster: analysis, interpretation of data, preparation of manuscript.

Sponsor's Role: Johns Hopkins HealthCare provided support for this research but had no role in the design, methods, subject recruitment, collection of survey data, data analysis, or preparation of the manuscript.

\section{REFERENCES}

1. Wolff JL, Starfield B, Anderson G. Prevalence, expenditures, and complications of multiple chronic conditions in the elderly. Arch Intern Med 2002;162:2269-2276.

2. Rothman AA, Wagner EH. Chronic illness management: What is the role of primary care? Ann Intern Med 2003;138:256-261.

3. Walter LC, Davidowitz NP, Heineken PA et al. Pitfalls of converting practice guidelines into quality measures: Lessons learned from a VA performance measure. JAMA 2004;291:2466-2470.
4. Boyd CM, Darer J, Boult C et al. Clinical practice guidelines and quality of care for older patients with multiple comorbid diseases: Implications for pay for performance. JAMA 2005;294:716-724.

5. Anderson G, Knickman JR. Changing the chronic care system to meet people's needs. Health Aff (Millwood) 2001;20:146-160.

6. Blendon RJ, Schoen C, DesRoches $\mathrm{C}$ et al. Common concerns amid diverse systems: Health care experiences in five countries. Health Aff (Millwood) 2003;22:106-121.

7. Weiner JP, Abrams C, Bodycombe D.The Johns Hopkins ACG Case-Mix System, Version 6 release notes. Section 2. The ACG Predictive Model: Helping to Manage Persons at Risk for High Future Costs [on-line]. Johns Hopkins University School of Public Health. Available at www.acg.jhsph.edu Accessed January 10, 2004.

8. Safran DG, Kosinski M, Tarlov AR et al. The Primary Care Assessment Survey: Tests of data quality and measurement performance. Med Care 1998;36: 728-739.

9. Institute of Medicine. Primary Care: America's Health in a New Era. Washington, DC: National Academy Press, 1996.

10. Montgomery JE, Irish JT, Wilson IB et al. Primary care experiences of Medicare beneficiaries, 1998-2000. J Gen Intern Med 2004;19:991-998.

11. Canadian College of Physicians. Relationship between family physicians and specialists/consultants in the provision of patient care: Report of a joint task force of the College of Family Physicians of Canada and the Royal College of Physicians and Surgeons of Canada. Can Fam Physician 1993;39:1309-1312.

12. Katz S, Ford AB, Moskowitz RW et al. Studies of illness in the aged. The index of ADL. A standardized measure of biological and psychological function. JAMA 1963;185:914-919.

13. Lawton MP, Brody EM. Assessment of older people: Self-maintaining and instrumental activities of daily living. Gerontologist 1969;9:179-186.

14. Lee Y, Kasper JD. Assessment of medical care by elderly people: General satisfaction and physician quality. Health Serv Res 1998;32:741-758.

15. Iezzoni LI, Davis RB, Soukup J et al. Satisfaction with quality and access to health care among people with disabling conditions. Int J Qual Health Care 2002;14:369-381.

16. Iezzoni LI, Davis RB, Soukup J et al. Quality dimensions that most concern people with physical and sensory disabilities. Arch Intern Med 2003;163: 2085-2092.

17. Hall JA, Milburn MA, Epstein AM. A causal model of health status and satisfaction with medical care. Med Care 1993;31:84-94.

18. Safran DG, Taira DA, Rogers WH et al. Linking primary care performance to outcomes of care. J Fam Pract 1998;47:213-220.

19. Parchman ML, Burge SK. The patient-physician relationship, primary care attributes, and preventive services. Fam Med 2004;36:22-27. 